\title{
Research of Oxidative Stress and Serum Thiols as a Criterion for the Antioxidant Barrier in Patients with Heart Failure (NYHA FC III-IV)
}

\author{
Mariana Georgieva Yordanova ${ }^{1,2 *}$ \\ ${ }^{1}$ Military Medical Academy, Multiprofile Hospital for Active Treatment, Bulgaria \\ ${ }^{2}$ Department of General Medicine and Clinical Laboratory, Medical University of Varna, Bulgaria
}

*Corresponding author: Mariana Georgieva Yordanova, Military Medical Academy, Multiprofile Hospital for Active Treatment, Varna, Bulgaria.

Received Date: April 30, 2020

Published Date: May 11, 2020

\section{Abstract}

Aim: To evaluate the status of oxidative stress and antioxidant systems (thiol and biological plasma antioxidant potential) in patients with heart failure, combined with disorders of carbohydrate metabolism and renal failure, and to what extent their values depend on the duration and severity of the disease (by NYHA).

Materials and Methods: Forty subjects (age 66.4 \pm 6.8 ) admitted for exacerbated chronic heart failure (III and IV FC), and 12 of them were examined in the same age range without clinical and echocardiography for HF. The levels of total thiols (SHp), reactive oxygen metabolites (dROMs), plasma antioxidant potential (BAP), creatinine, calculated GFR, glucose and C-reactive protein are analysed. Measurement of SHp, dROMs and BAP assays was performed spectrophotometrically with kits of Diacron International-Italy with an Olympus AU400 biochemical analyser and Carpe diam analyser (Diacron).

Results: An increase in oxidative stress was observed in the patient group, which had a high negative correlation with the thiol groups and the plasma antioxidant potential ( $\mathrm{r}=-0.7404 ; \mathrm{r}=-0.7567)$. The decrease in glomerular filtration also corresponds to a high degree with the reduction of the organism's antioxidant capacity and the increase of oxidative stress. We found a high degree of dependence on the severity of HF (according to the NYHA classification), with the studied levels of oxidative stress and the antioxidant barrier. A similar relationship is observed between disease duration and SHp, dROMs and BAP concentrations.

Conclusion: Oxidative stress plays an essential role in the pathogenesis and progression of CHF. The observed thiol levels have decreased statistically significant, such as the collapse of the antioxidant system is key in the disturbed homeostasis of organisms in ROS/RNS coping.

Keywords: Thiols; Oxidative stress; Antioxidant system; Heart failure; GFR, Inflammatory response.

\section{Introduction}

Heart failure is a severe chronic condition where, as a result of dysfunction, the heart is unable to expel sufficient blood to meet the body's metabolic needs. The most common causes are coronary heart disease, myocardial infarction, congenital heart defects, damage to the heart valves, atrial fibrillation. Heart failure (HF) is the most common cause of hospitalisation in people over 65 and is associated with increased health costs. It is a global pandemic affecting at least 26 million people worldwide, with disease rates increasing as the population ages [1]. The death rate from this diagnosis in Bulgaria has doubled, with over 20,000 people dying annually. Oxidative stress is a key element in the pathophysiology of heart failure (HF) [2]. Several studies support the hypothesis of a link between oxidative status change and the development and progression of many diseases, such as neurodegenerative conditions, cardiovascular, inflammatory diseases, and cancer [2-5]. In the course of oxidation processes, reactive free radicals (ROS) are formed continuously, which are missing one electron. To achieve their stability as compounds, they obtain electrons from 
other organic molecules such as DNA, fats, proteins, and enzymes by oxidising them. Free radicals are extremely reactive, damaging cell membranes and DNA molecules [6] (Figure 1).

\section{oxidative stress}

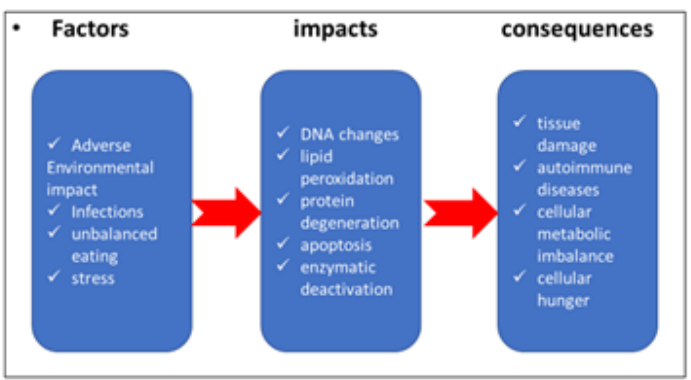

Figure 1: Factors contributing to oxidative stress and its effects in aerobic organisms.

Physiologically free radicals are formed during normal metabolic processes in the cell. They play a role in the regulation of basic functions and act as signalling molecules in the transduction cascades or pathways for various growth factors, cytokines and hormones [6]. On the other hand, the so-called "oxidative explosion" is absolutely necessary for the initiation of immunological responses. It activates neutrophil cells and phagocytosis as the body's primary immune response against foreign antigens. Oxidative stress is a term used to refer to an imbalance between the concentrations of reactive oxygen and nitrogen species (RNS) formed and the body's antioxidant protection. The body counteracts oxidative stress with its plasma and intracellular antioxidants. An antioxidant is any substance or compound that can bind ROS and RNS or inhibit the oxidation process in the cell. The deficiency of natural antioxidants increases with advancing age and the worsening of chronic pathological processes.

Thiols are compounds with a free sulfhydryl (R-SH) group. Most often they are presented in the form of proteins containing one or more free cysteine groups, or low molecular weight compounds (e.g. glutathione GSH). They are localised in cells and extracellular fluids. They are endogenous organic compounds that support the maintenance of aerobic cells in a reducing state despite the oxidising environment. R-SH exists in vivo in three forms, which include free thiol (e.g., cysteine), identical thiols formed between two homodisulfides (e.g., GSSG glutathione disulfide) and heterodisulfides formed between different thiols (e.g. proteinbound thiols). Proteins with their sulfhydryl groups are the main component of the serum antioxidant effect. In the case of high oxidative stress, thiol levels are reduced to neutralise reactive oxygen species. In this case, the sulfhydryl groups of the thiols are oxidised to form disulfide (-S-S-) bonds. These connections can be transformed back to thiols, and thus the thiol/disulfide homeostasis is maintained [7]. Thiol is one of the most important antioxidant barriers in humans, and thiol / disulfide homeostasis is a new marker for oxidative stress [8]. In a recent study, SH protein groups were found to contribute $52.9 \%$ of measured total serum antioxidant capacity in healthy subjects [9]. Impaired balance oxidative stress, antioxidant defences and changes occurring in the redox thiol system are associated with ischemic heart disease, atherosclerosis, renal and hepatic failure, rheumatoid arthritis, dementia and Alzheimer's disease. In addition to their role in protecting against free radicals, thiols share a significant role in detoxification, signal transduction, apoptosis, and various other molecular-level functions.

Frenay A-RS, et al. shows that high serum concentrations of $\mathrm{R}-\mathrm{SH}$ are associated with a favourable cardiovascular risk profile and better survival for patients with the transplanted kidney [10]. Oxidative stress causes the development of myocardial and vascular dysfunction and is an important pathophysiological factor in HF. Quantitative studies of reactive oxygen metabolites (dROMs), biological antioxidant potential (BAP), and the main aminothiol compounds in plasma or serum stimulate scientific search with the potential for therapeutic modulation.

\section{Materials and Methods}

Forty patients ( 24 men and 16 women) that have a mean age of 66.38 years (range 54-78 years) admitted to the MMAVarna Cardiology Department for exacerbated CHF (NYHA IIIIV FC) were examined. Criteria for inclusion in the study are the presence of typical subjective and objective symptoms of heart failure and ultrasound-proven ventricular dysfunction (ejection fraction $<35 \%$ ). The control group consisted of 12 individuals with a mean age of 62.5 years (range 55-70 years) with no subjective and objective data on cardiovascular disease, no clinical and laboratory criteria for diabetes and impaired renal function. The study was approved by the Ethics Committee of MMA-Varna. Body mass index (BMI) was determined in both groups. A standard pre-analytical procedure is used to take venous blood. The blood serum was removed by centrifugation for 10 minutes at $3500 \mathrm{rpm}$. In serum, we determine the following indicators: creatinine, CRP, glucose, cholesterol, oxidative stress (dROMs), serum antioxidant capacity (BAP), and total serum thiol. GFR was calculated based on serum creatinine using the MDRD formula. Oxidative stress (dROMs) and serum antioxidant capacity (BAP) were determined spectrophotometrically with ready-made kits from Diacron International-Italy using a semi-automatic Carpe diam analyser (Diacron Labs Italy). The d-ROM test is based on the ability of a biological sample to oxidise aromatic amine (DPPD). Reactive oxygen metabolites (ROMs) (hydroperoxides, chloramines and their derivatives) react with appropriately buffered chromogen and develop a colour derivative that is photometric at $505 \mathrm{~nm}$. The concentration of this class of ROMs directly correlates with the intensity of the colour and the increased levels of oxidative stress. The units that express dROMs are Carratelli units ( 1 CARR $U=0.08$ $\mathrm{mg} \%$ hydrogen peroxide). The range in healthy individuals is 250 300 UCARR

The BAP test is spectrophotometric $(\lambda=505 \mathrm{~nm})$ for measuring the antioxidant capacity (AOC) of a sample placed in a solution of 
ferric chloride. It has the ability to reduce iron from ferric Fe3+ to Ferro Fe2+ ion. Reducing the intensity of the colour is a criterion for the concentration of antioxidants present in the plasma. For both tests, quality control is performed with two levels of control materials (Low and High).

The testing of plasma thiol groups (R-SH) (lipoic acid, glutathione, cysteine) is performed with a colourimetric SHp test (Diacron International-Italy) with an Olympus AI 400 biochemical analyser with our adapted program. The principle of the method is based on the interaction of thiols with 5, 5'-dithiobis(2-nitrobenzoic acid) (DTNB), forming highly coloured anions with maximum absorption at $405 \mathrm{~nm}$. Thiols are extremely unstable. Within the same day, samples were examined in fresh plasma (serum) without a trace of hemolysis. Pre-analytic requirements have been described and imposed because time and oxygen from the air enhances the reduction of glutathione by $\gamma$-glutamyl cysteine

\section{Results and Discussion}

Table 1: Clinical, demographic, and biochemical characteristics.

\begin{tabular}{|c|c|c|}
\hline Parameters & Patient group $(n=40)$ & control group $(n=12)$ \\
\hline Age (years) & $66.4 \pm 6.8$ & $62.5 \pm 5.2$ \\
\hline $\begin{array}{c}\text { Gender } \\
\text { Men } \\
\text { women }\end{array}$ & $\begin{array}{l}24 \\
16\end{array}$ & $\begin{array}{l}7 \\
5\end{array}$ \\
\hline $\begin{array}{l}\text { NYHA Classification } \\
\text { Class III } \\
\text { Class IV }\end{array}$ & $\begin{array}{l}18 \\
22\end{array}$ & \\
\hline duration of the disease & $5.0 \pm 1.8$ & \\
\hline up to 2 year & $\mathrm{n}=10$ & \\
\hline up to 5 year & $\mathrm{n}=13$ & \\
\hline up to 8 year & $\mathrm{n}=17$ & \\
\hline *BMI (body weight/height2) & $26.4 \pm 5.8$ & \\
\hline${ }^{* *}$ Glucose $(\mathrm{mmol} / \mathrm{l})$ & $8.16 \pm 2.71$ & $6.36 \pm 0.80$ \\
\hline${ }^{* * *}$ Cholesterol (mmol/l) & $6.88 \pm$ & $6.65 \pm$ \\
\hline $\mathrm{RR} \mathrm{mm} / \mathrm{Hg}$ & $145 \pm 27 / 96 \pm 10$ & $136 \pm 19 / 88 \pm 12$ \\
\hline
\end{tabular}

and the rapid conversion of reduced glutathione into oxidised form, which interferes with the result. Each series was controlled by two levels of controls - low and high. VK 2.8\%; 5.2\%, bis $2.8 \%$ respectively for low control and high. CRP and serum creatinine were determined using routine methods of an Olympus AU 640 biochemical analyser with Beckman Coulter reagents (USA).

\section{Statistical Methods}

Data analysis is performed with Graph Pad Prism v software. 6.0 using standard statistical methods (descriptive statistics, nonparametric T-test for mean comparison, ANOVA test for continuous variables with normal distribution between the groups of analysis, variance and Pearson correlation analysis). Biochemical parametric data were presented as mean and standard deviation (mean \pm SD) at a 95\% confidence interval. Statistical significance was stated at $\mathrm{p}<0.05$.

normal range BMI (18.50-25)

**normal range Glucose 3.5-6.0 mmol//

***normal range Cholesterol $<5.17 \mathrm{mmol} / \mathrm{l}$

Clinical, demographic and biochemical characteristics of the study population are described in Table 1. Hypertension, dyslipidemia, diabetes mellitus are major risk factors for the development of heart failure. Nevertheless, obesity, hypodynamia, smoking, genetic predisposition also play an important role in its etiopathogenesis. CHF and CKD share common etiologies, with dynamic and bidirectional interactions between the cardiovascular system and the renal can explain the occurrence of simultaneous organ dysfunction [11]. Oxidative stress has been identified as a unifying mechanism in the pathogenesis of CKD and CVD [12]. Hyperglycemia promotes a state of oxidative stress that increases insulin resistance and $\beta$-cell dysfunction. ROS play a major role in the pathogenesis of microvascular and macrovascular complications in diabetes.

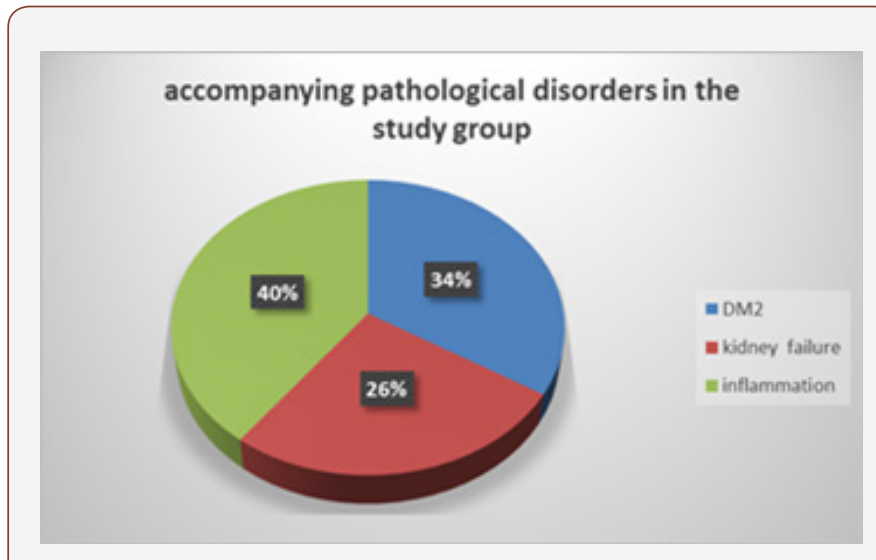

Figure 2: Presence of concomitant diseases in the background of chronic heart failure. 
Figure 2 shows the concomitant diseases (KDIGO 2012 and DM renal failure) imposed by HF that increase the risk and severity of the disease. Patients had one or two concomitant diseases. The values of the studied indicators of oxidative stress in the patient group were statistically significantly higher, and for antioxidant protection lower than in the control group. In patients with HF, we have seen that as oxidative stress increases, antioxidant protection and total thiols progressively decrease. There is a high negative correlation between dROMs levels with those of SHp and BAP. Impaired renal function, inflammatory response and impaired hemodynamics with ventricular disease are factors that generate many reactive oxygen species (Table $2 \& 3$ ).

Table 2: Values of the investigated parameters in the patients and control group.

\begin{tabular}{|c|c|c|}
\hline Parameters & Patient group $(\mathbf{n}=\mathbf{4 0})$ & $\begin{array}{c}\text { control group } \\
(\mathbf{n = 1 2})\end{array}$ \\
\hline${ }^{*}$ dROMs UCarr & $504.53 \pm 117.1$ & $346.58 \pm 51.9$ \\
\hline${ }^{* * \text { BAP test umol/l }}$ & $1996 \pm 252.1$ & $2420 \pm 255.9$ \\
\hline $\begin{array}{c}{ }^{* * *} \text { Shp test (thiols) } \\
\text { umol/l }\end{array}$ & $398.0 \pm 89.5$ & $585.58 \pm 45.4$ \\
\hline $\begin{array}{c}\text { Creatinin (umol/l) } \\
\text { ****GFR ml/min/1.73 } \\
\mathrm{m} 2\end{array}$ & $176.75 \pm 98.4$ & $112.92 \pm 15.6$ \\
\hline CRP mg/l & $61.25 \pm 18.6$ & $64.3 \pm 6.5$ \\
\hline
\end{tabular}

${ }^{*} \mathrm{~d}-\mathrm{ROMs}$ is a patented test by Dicarbon International sas, Grosseto (Italy; $1 \mathrm{UCarr}$ equals $0.08 \mathrm{mg} / \mathrm{dL}$ of $\mathrm{H} 2 \mathrm{O} 2$. The range in healthy peoples is 250-300 U CARR. Increased values directly correlate to increased levels of oxidative stress.

**The reference values of normal level of antioxidant defense is $>2200$ $\mu \mathrm{mol} / \mathrm{L}$ (reference iron -reducing agent: Vitamin C).

***Shp test The reference values of normal level 450-650 мкмол/л.

**** calculated using the Modification of Diet in Renal Disease formula

Table 3: Correlation between oxidative stress markers (dROMs) and antioxidant protection (with SHp and BAP).

\begin{tabular}{|c|c|c|}
\hline \multirow{2}{*}{ Correlation Pearson $\mathbf{r}$} & $\begin{array}{c}\text { dROMs } \\
\text { vs. }\end{array}$ & $\begin{array}{c}\text { dROMs } \\
\text { vs. }\end{array}$ \\
& SHp & -0.7567 \\
\hline $\mathrm{r}$ & -0.7404 & 0.5726 \\
\hline $95 \%$ confidence interval & -0.8548 to -0.5573 & -0.8645 to -0.5824 \\
\hline R square & 0.5481 & \\
\hline P value & & $<0.0001$ \\
\hline P (two-tailed) & $<0.0001$ & \\
\hline
\end{tabular}

ROS and RNS stimulate cell damage and functional pathways in them [11]. The links between obesity, inflammation, dyslipidemia, CKD and CVD are manifested through the metabolic syndrome. The patient group was divided into 4 subgroups depending on the degree of renal dysfunction and the GFR values of $\mathrm{ml} / \mathrm{min} / 1.73 \mathrm{~m} 2$. (over $60(n=7) ; 40-60(n=15) ; 20-40(n=10)$ and below $20(n=8)$. Comparative analysis showed statistically significant $(\mathrm{p}<0.001)$ increase in oxidative stress ( $\mathrm{dROMs}$ ) in proportion to a decrease in glomerular filtration tissue. Tissue hypoxia leads to an imbalance in mitochondrial electron transport and increased oxidative stress, intracellular $\mathrm{Ca} 2$ + overload and cell death $[13,14]$. Ventricular dysfunction and impaired renal perfusion lead to a severe decrease in glomerular filtration rate (GFR) and impaired renal excretory function. Nephrosclerosis, as a result of long-running arterial hypertension, diabetes, and progressive atherosclerosis, reduce the kidney's functional capacity. Free radicals are assumed to cause a change in normal membrane fluidity of cells, alter her permeability and diffusion of oxygen [6]. The antioxidant capacity and thiol groups respectively decline with decreasing GFR (Figure 3). Thiols are crucial active components of antioxidant capacity. Serum thiol depletion or oxidation in patients with cardiovascular disease (CVD), including acute myocardial infarction, has been reported compared to controls [15]. S-glutathionylated proteins accumulate under conditions of oxidative/nitrosative stress. They can easily be reduced to free thiol groups when normal cellular redox status is restored by glutaredoxins (thioltransferases) or reducing agents [16]. However, under the conditions of pathophysiological processes the ratio GSH: GSSG is reduced. When GSSG is accumulated in cells, it can undergo disulfide exchange reactions with protein thiols, leading to their S-glutathionylation [17]. We found a significant negative correlation between impaired renal function expressed by GFR and dROMs values, as well as a significant positive correlation with BAP and R-SH tests (Figure 3 \& Table 4). Our study confirms the studies by Shetty JK, et al. who used creatinine to correlate thiol levels and with the severity of renal failure [18-19].

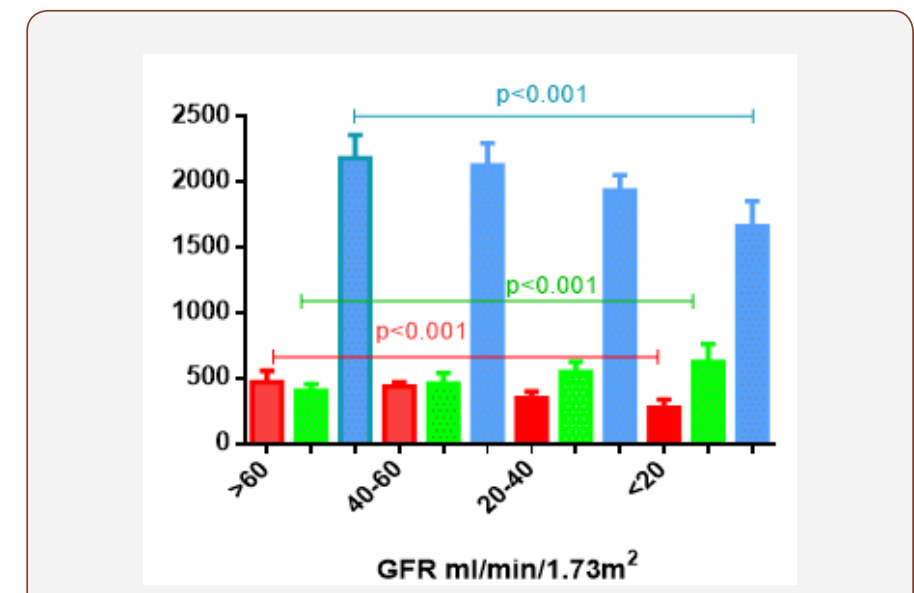

Figure 3: Comparative analysis of GFR to oxidative stress and antioxidant markers values.

Anne M Koning, et al. in their studies suggest that higher levels of free serum thiols are associated with favorable disease outcome, i.e decreased rates of rehospitalization and increased survival in stable CHF patients [15]. Protein -SH groups interrupt the lipid peroxidation reaction with peroxyl radicals during the first steps of oxidation reactions. Chronic inflammation is involved in the pathogenesis of cardiovascular disease, diabetes, obesity, atherosclerosis. Inflammatory processes with neutrophil activation are a major source of multiple ROS and lead to oxidative stress [20]. Several oxidized proteins, glycated products and lipid peroxides are produced, an increase in homocysteine that leads to degenerative changes in vascular endothelial cells. Intracellular Thiol (GSH: GSSG) homeostasis disorder results in an imbalanced immune response, inflammation and increased susceptibility to infection. Oxidative stress generates high levels of inflammatory cytokines, 
such as TNF- $\alpha$ and interleukin-6 (IL-6), and adhesion molecules [21]. The correlation analysis made, showed that the degree of inflammatory response was inversely related to the levels of thiols and, accordingly to the reduction of the overall antioxidant status. There is a significant negative correlation between the degree of inflammatory response with the markers of antioxidant protection (SHp and BAP test), whereas, with oxidative stress, this dependence is moderate (Table 4).

Table 4: Correlation between markers for oxidative stress and antioxidant protection with CRP protein.

\begin{tabular}{|c|c|c|c|}
\hline \multirow{2}{*}{ Correlation Pearson r } & CRP & CRP & CRP \\
& vs. & vs. & vs. \\
& dROMs & BAP \\
\hline $\mathrm{r}$ & -0.5127 & 0.3618 & -0.467 \\
\hline \multirow{2}{*}{ 95\% confidence interval } & -0.7107 to & 0.05663 to & -0.6797 to \\
& -0.2393 & 0.6052 & -0.1819 \\
\hline R square & 0.2628 & 0.1309 & 0.2181 \\
\hline P value & & & \\
\hline P (two-tailed) & 0.0007 & 0.0218 & 0.0024 \\
\hline
\end{tabular}

In our study, we found that free thiol values decreased significantly with disease duration, with this dependence being statistically significant (Figure 4). Anne M. Koning suggests a favorable association of free thiols with HF severity markers and prognosis. Patients who had above-average serum thiols were younger, with better renal function and had lower NT-proBNP levels [15]. In patients with CHF, we find a high positive relationship between elevated concentrations of dROMs and NYHA functional class (Table 5). Identical observations have been reported by Ewa Romuk et al., who use MDA as a marker for oxidative stress (lipid peroxidation) [13]. Thiol values and antioxidant capacity are inversely proportional to the increase in oxidative stress and the severity of the disease. We observe a high negative correlation between NYHA functional class with serum thiols and plasma antioxidant potential. Evidently, reducing antioxidant systems and mechanisms are critical to the progress of oxidative damage and the progression of CHF. In previous studies, Anne M. Koning et al. have also reported a significant decrease in serum protein thiols in active diseases, including CVD and CHF [15,22,23].

Our study confirms the evidence of a strong reduction in thiols and a reduction in the antioxidant potential of plasma in patients admitted with an exacerbation of heart failure. The values of these indicators decrease with progression of the disease and FC of NYHA HF. The disadvantage of the New York Heart Association (NYHA) Classification is that it is based on the assessment of the severity of the patient's symptoms and involves a high degree of subjectivity. The functional class of a patient with HF may fluctuate for short periods, especially in periods of decompensation. The correlation with the level of ventricular dysfunction and the prognosis to patients is low. Our patient group is not very large. It should be expanded with NYHA FC I-II patients, which can better assess the tendency for increased oxidative stress and reduced antioxidants.

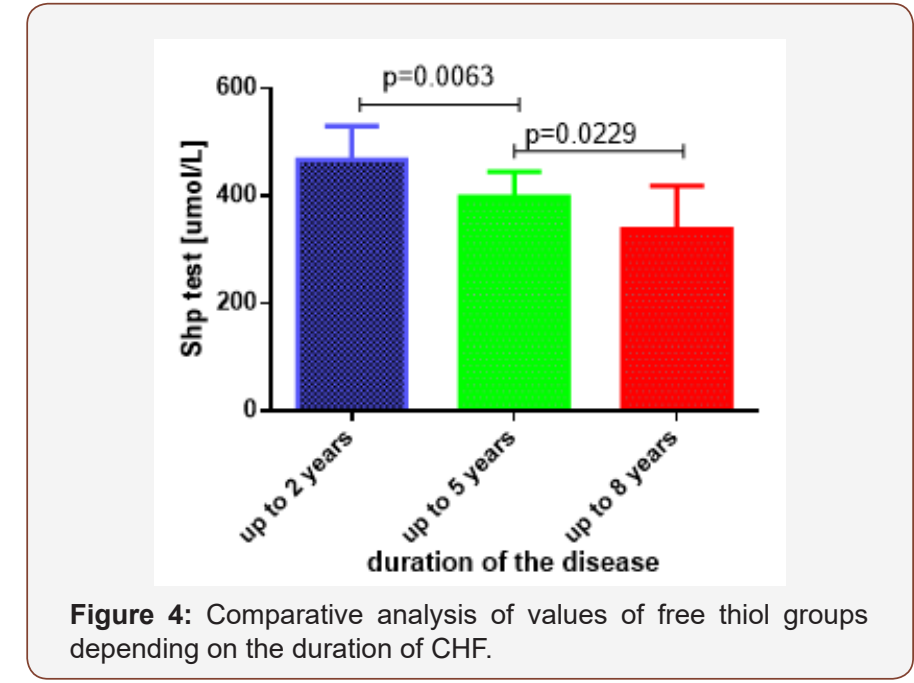

Table 5: Correlation between NYHA FC and oxidative stress and antioxidant indices.

\begin{tabular}{|c|c|c|c|}
\hline \multirow{2}{*}{ Correlation Pearson $\mathbf{r}$} & $\begin{array}{c}\text { NYHA class } \\
\text { vs } \\
\text { SHp test }\end{array}$ & $\begin{array}{c}\text { NYHA class } \\
\text { vs } \\
\text { dROMs }\end{array}$ & $\begin{array}{c}\text { NYHA class } \\
\text { vs }\end{array}$ \\
\hline r & -0.7504 & 0.662 & -0.6677 \\
\hline \multirow{2}{*}{$95 \%$ confidence interval } & -0.8607 to & 0.4415 to & -0.8106 to \\
& -0.5727 & 0.8071 & -0.4496 \\
\hline R square & 0.5631 & 0.4383 & 0.4458 \\
\hline P value & & & \\
\hline P (two-tailed) & $<0.0001$ & $<0.0001$ & $<0.0001$ \\
\hline
\end{tabular}

\section{Conclusion}

Oxidative stress, as an important element in the pathophysiology of CHF, contributes to the development of myocardial and vascular dysfunction. The observed statistically significant decrease in thiol levels, such as the collapse of the antioxidant system, are key in the disrupted homeostasis of organisms in ROS/RNS coping. The more pathophysiological disorders involved in the disease process, the greater the decrease in total thiols. CVD and CKD are closely related diseases with increasing morbidity and mortality. Prevention and treatment of these diseases are major goals in healthcare systems worldwide. Research on antioxidant systems may be useful for therapeutic purposes and management.

\section{Acknowledgement}

None.

\section{Conflict of Interest}

No conflict of interest.

\section{References}

1. Savarese G, Lund LH (2017) Global Public Health Burden of Heart Failure. Card Fail Rev 3(1): 7-11.

2. Koning A (2017) Exploring Redox Biology in physiology and disease. Rijksuniversiteit Groningen pp. 275.

3. Brunelli E, La Russa D, Pellegrino D (2017) Impaired Oxidative Status Is Strongly Associated with Cardiovascular Risk Factors. Oxid Med Cell Longev 2017: 6480145. 
4. Pisoschi AM, Pop A (2015) The role of antioxidants in the chemistry of oxidative stress: a review. European Journal of Medicinal Chemistry 97: 55-74.

5. Montezano AC, Dulak-Lis M, Tsiropoulou S, Harvey A, Briones AM, et al (2015) Oxidative stress and human hypertension: vascular mechanisms, biomarkers, and novel therapies. Can J Cardiol 31(5): 631-641.

6. Petersen RC (2017) Free-radicals and advanced chemistries involved in cell membrane organization influence oxygen diffusion and pathology treatment. AIMS Biophys 4(2):240-283.

7. Schillern EEM, Pasch A, Feelisch M, Waanders F, Hendriks SH, et al (2019) Serum free thiols in type 2 diabetes mellitus: A prospective study. J Clin Transl Endocrinol 16: 100182.

8. Altıparmak IH, Erkuş ME, Sezen H, Demirbag R, Gunebakmaz O, et al (2016) The relation of serum thiol levels and thiol/disulphide homeostasis with the severity of coronary artery disease. Kardiol Pol 74(11) 1346-1353.

9. Kalkan G, Emre S, Alisik M, Aktaş A, Baran P (2019) Dynamic thiol/disulfide homeostasis in patients with lichen planus J Clin Lab Anal 33(1): e22642.

10. Frenay AS, de Borst MH, Bachtler M, Tschopp N, Keyzer CA, et al. (2016) Serum free sulfhydryl status is associated with patient and graft survival in0000000000+ renal transplant recipients. Free Radic Biol Med 99: 345-351.

11. David M Small, Glenda C Gobe (2012) Oxidative Stress and Antioxidan Therapy in Chronic Kidney and Cardiovascular Disease.

12. Bongartz LG, Cramer MJ, Doevendans PA, Joles JA, Braam B (2005) The severe cardiorenal syndrome: 'Guyton revisited'. Eur Heart J 26(1): 1117.

13. Romuk E, Wojciechowska C, Jacheć W (2019) Comparison of Oxidative Stress Parameters in Heart Failure Patients Depending on Ischaemic or Nonischaemic Aetiology. Oxid Med Cell Longev 2019: 7156038.
14. M Seddon, YH Looi, AM Shah (2007) Oxidative stress and redox signalling in cardiac hypertrophy and heart failure. Heart 93(8): 903-907.

15. Koning AM, Meijers WC, Pasch A, Leuvenink HGD, Frenay AS, et al. (2016) Serum free thiols in chronic heart failure. Pharmacol Res 111: 452-458.

16. Prakash M, Shetty MS, Tilak P (2009) Total Thiols: Biomedical Importance And Their Alteration In Various Disorders. Online J Health Allied Scs 8(2): 2.

17. Konarkowska B, Aitken JF, Kistler J, Zhang S, Cooper GJ (2005) Thiol reducing compounds prevent human amylin-evoked cytotoxicity. FEBS J 272: 4949-4959.

18. Shetty JK, Prakash M, Tripathy S (2007) Serum Paraoxonase activity and protein thiols in chronic renal failure patients. Asian J Biochem 2: 274 278.

19. Himmelfarb J, McMonagle E, Freedman S, Klenzak J, McMenamin E, et al. (2004) Oxidative stress is increased in critically ill patients with acute renal failure. J Am Soc Nephrol 15: 2449-2456.

20. Van Campenhout A, Van Campenhout C, Lagrou AR, Abrams P, Moorkens $G$, et al. (2006) Impact of diabetes mellitus on the relationships between iron-, inflammatory-and oxidative stress status. Diabetes Metab Res Rev 22: 444-454.

21. P Ghezzi (2011) Role of glutathione in immunity and inflammation in the lung. International Journal of General Medicine 4: 105-113.

22. Banne AF, Amiri A, Pero RW (2003) Reduced level of serum thiols in patients with a diagnosis of active disease. J Anti-Aging Med 6(4): 327-334.

23. Kundi H, Ates I, Kiziltunc E, Cetin M, Cicekcioglu H, et al. (2015) A novel oxidative stress marker in acute myocardial infarction; thiol/disulphide homeostasis. Am J Emerg Med 33(11): 1567-1571. 